\title{
Fundamental Finite Key Limits for Information Reconciliation in Quantum Key Distribution
}

\author{
Marco Tomamichel*, Jesus Martinez-Mateo ${ }^{\dagger}$, Christoph Pacher $^{\ddagger}$, David Elkouss ${ }^{\S}$ \\ *Centre for Quantum Technologies, National University of Singapore \\ Email: cqtmarco@nus.edu.sg \\ ${ }^{\dagger}$ Facultad de Informática, Universidad Politécnica de Madrid \\ $\ddagger$ Department of Safety \& Security, AIT Austrian Institute of Technology \\ $\S$ Departamento de Analisis Matemático, Universidad Complutense de Madrid
}

\begin{abstract}
The security of quantum key distribution protocols is guaranteed by the laws of quantum mechanics. However, a precise analysis of the security properties requires tools from both classical cryptography and information theory. Here, we employ recent results in non-asymptotic classical information theory to show that information reconciliation imposes fundamental limitations on the amount of secret key that can be extracted in the finite key regime. In particular, we find that an often used approximation for the information leakage during one-way information reconciliation is flawed and we propose an improved estimate.
\end{abstract}

\section{INTRODUCTION}

Quantum key distribution (QKD) [3], [8] is a prime example of the interdisciplinary nature of quantum cryptography and the first application of quantum science that matured into the realm of engineering and commercial development. While the security of the generated key is intuitively guaranteed by the laws of quantum mechanics, a precise analysis of the security requires tools from both classical cryptography and information theory (see [17], [25] for early security proofs and [23] for a comprehensive review). This is particularly relevant when investigating the security of QKD in a practical setting where the resources available to the honest parties are finite and the security analysis consequently relies on nonasymptotic information theory.

In the following, we consider QKD protocols between two honest parties, Alice and Bob, which can be partitioned into the following rough steps. In the quantum phase, $N$ physical systems are prepared, exchanged and measured by Alice and Bob. In the parameter estimation (PE) phase, relevant parameters describing the channel between Alice and Bob are estimated from correlations measured in the quantum phase. If the estimated parameters do not allow extraction of a secure key, the protocol aborts at this point. Otherwise, the remaining measurement data is condensed into two highly correlated bit strings of length $n$ in the sifting phase - the raw keys $X^{n}$ for Alice and $Y^{n}$ for Bob. We call $n$ the block length and it is the quantity that is usually limited by practical considerations (time interval between generated keys, amount of key that has to be discarded in case Alice and Bob create different keys, hardware restrictions). In the information reconciliation (IR) phase, Alice and Bob exchange classical information about $X^{n}$ over a public channel in order for Bob to compute an estimate $\hat{X}^{n}$ of $X^{n}$. The confirmation (CO) phase ensures that $\hat{X}^{n}=X^{n}$ holds with high probability or aborts the protocol. Finally, in the privacy amplification (PA) phase, Alice and Bob distill a shared secret key of $\ell$ bits from $X^{n}$ and $\hat{X}^{n}$. We say that a protocol is secure if (up to some error tolerance) both Alice and Bob hold an identical, uniform key that is independent of the information gathered by an eavesdropper during the protocol, for any eavesdropper with access to the quantum and the authenticated classical channel.

The ratio $\ell / N$ is constrained by the following effects: 1) Some measurement results are published for PE and subsequently discarded. 2) The sifting phase removes data that is not expected to be highly correlated, thus further reducing the length $n$ of the raw key. 3) Additional information about the raw keys is leaked to the eavesdropper during the IR and CO phase. 4) To remove correlations with the eavesdropper, $X^{n}$ and $\hat{X}^{n}$ need to be purged in the PA phase, resulting in a shorter key. Some of these contributions vanish asymptotically for large $N$ while others approach fundamental limits. ${ }^{1}$

Modern tools allow to analyze QKD protocols that are secure against the most general attacks. They provide lower bounds on the number of secure key bits that can be extracted for a fixed block length, $n$. For the BB84 protocol, such proofs are for example given in [22], [24] and [9]. These proofs were subsequently simplified to achieve better key rates in [31] and [12], respectively. All results have in common that the key rate that can be achieved with finite resources is strictly smaller than the asymptotic limit for large $n$ - as one would intuitively expect.

We are concerned with a complementary question: Given a secure but otherwise arbitrary QKD protocol for a fixed $n$, are there fundamental upper bounds on the length of the key that can be produced by this protocol? Such bounds are of theoretical as well as practical interest since they provide a benchmark against which contemporary implementations of QKD can be measured. In the asymptotic regime of large block lengths, such upper bounds have already been investigated, for example in [19]. Here we limit the discussion to IR and focus on bounds that solely arise due to finite block lengths

\footnotetext{
${ }^{1}$ Consider, for example, BB84 with asymmetric basis choice [15] on a channel with quantum bit error rate $Q$. There, contributions 1 ) and 2) vanish asymptotically while contributions 3 ) and 4) converge to $h(Q)$.
} 
(Sec. II). We complement the bounds with a numerical study of achievable leak values with LDPC codes (Sec. III), and study some possible improvements and open issues (Sec. IV).

\section{FUNDAMENTAL LIMITS FOR RECONCILIATION}

We consider one-way IR protocols, where Alice first computes a syndrome, $M \in \mathcal{M}$, from her raw key, $X^{n}$, and sends it to Bob who uses the syndrome together with his own raw key, $Y^{n}$, to construct an estimate $\hat{X}^{n}$ of $X^{n}$. We are interested in the size of the syndrome (in bits), denoted $\log |\mathcal{M}|$, and the probability of error, $\operatorname{Pr}\left[X^{n} \neq \hat{X}^{n}\right]$. In most contemporary security proofs $\log |\mathcal{M}|$ enters the calculation of the key rate rather directly. ${ }^{2}$ More precisely, to achieve security it is necessary (but not sufficient) that

$$
\ell \leq n-\mathrm{leak}_{E C},
$$

where leak $k_{E C}$ is the amount of information leaked to the eavesdropper during IR. Since it is usually impossible to determine leak $_{E C}$ precisely, this term is often bounded as leak $_{E C} \leq \log |\mathcal{M}|$. In the following, we are thus interested in finding lower bounds on $\log |\mathcal{M}|$.

Let $P_{X Y}$ be a probability distribution. We say that an IR protocol is $\varepsilon$-correct on $P_{X Y}$ if it satisfies $\operatorname{Pr}\left[X^{n} \neq \hat{X}^{n}\right] \leq \varepsilon$ when $X^{n}$ and $Y^{n}$ are distributed according to $\left(P_{X Y}\right)^{\times n}$. Any such protocol (under weak conditions on $P_{X Y}$ and for small $\varepsilon$ ) satisfies $\frac{1}{n} \log |\mathcal{M}| \geq H(X \mid Y)_{P}$ [28]. Moreover, equality can be achieved for $n \rightarrow \infty$ [26]. On first sight, it thus appears reasonable to compare the performance of a finite block length protocol by comparing $\log |\mathcal{M}|$ with its asymptotic limit. In fact, for the purpose of numerical simulations, the amount of one-way communication from Alice to Bob required to perform IR is usually approximated as leak $\mathrm{k}_{E C} \approx \xi \cdot n H(X \mid Y)_{P}$, where $\xi>1$ is the reconciliation (error correction) efficiency. The constant $\xi$ is often chosen in the range $\xi=1.05$ to $\xi=1.2$. $^{2}$ However, this choice is scarcely motivated and independent of the block length, the bit error rate and the required correctness considered.

Here, we argue that this approximation is unnecessarily rough in light of recent progress in non-asymptotic information theory. Strassen [27] already observed in the context of noisy channel coding that the asymptotic expansion of the fundamental limit for large $n$ admits a Gaussian approximation. This approximation was recently refined by Polyanskiy et al. [21] (see also [11]). The problem of information reconciliation also called source compression with side information - was investigated by Hayashi [10] and recently by Tan and Kosut [28]. Here we go slightly beyond this and provide bounds on the asymptotic expansion up to third order:

Theorem 1. Let $0<\varepsilon<1$ and $P_{X Y}$ arbitrary. Then, for large $n$, any $\varepsilon$-correct IR protocol on $P_{X Y}$ satisfies

$$
\begin{aligned}
\log |\mathcal{M}| & \geq n H(X \mid Y)+\sqrt{n V(X \mid Y)} \Phi^{-1}(1-\varepsilon) \\
& -\frac{1}{2} \log n-O(1),
\end{aligned}
$$

\footnotetext{
${ }^{2}$ Recent works analyzing the finite block length behavior using this approximation include [1], [4], [6], [12], [14], [24], [31].
}
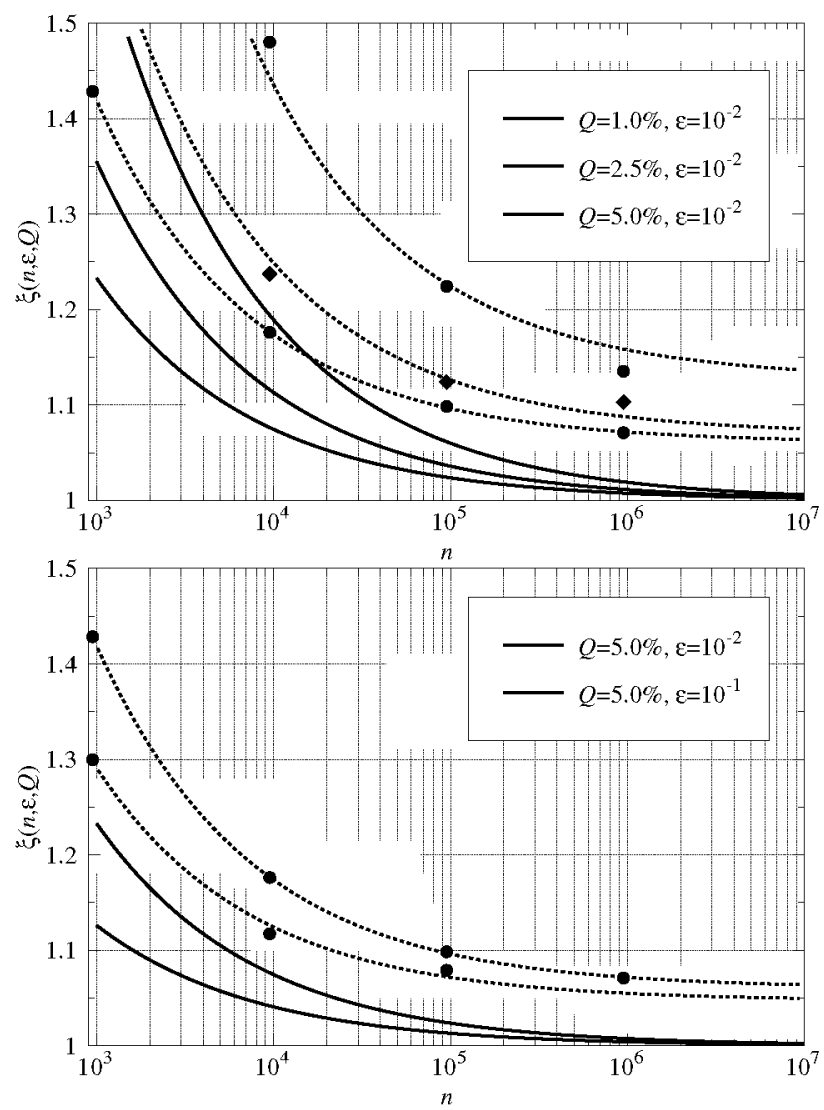

Fig. 1: The solid lines show the fundamental limit of the efficiency, $\xi(n, \varepsilon ; Q)$, as a function of $n$ for different values of $Q$ and $\varepsilon$. The dotted lines show fits (see Table I) to Eq. (4) for simulated LDPC codes (marked with symbols).

where $H(X \mid Y):=\operatorname{Exp}\left[\log \frac{P_{Y}}{P_{X Y}}\right]$ is the conditional entropy, $V(X \mid Y):=\operatorname{Var}\left[\log \frac{P_{Y}}{P_{X Y}}\right]$ is the conditional entropy variance, and $\Phi$ is the cumulative standard normal distribution. Moreover, there exists an $\varepsilon$-correct IR protocol with $\log |\mathcal{M}| \leq$ $n H(X \mid Y)+\sqrt{n V(X \mid Y)} \Phi^{-1}(1-\varepsilon)+\frac{1}{2} \log n+O(1)$.

The proof uses standard techniques, namely Yassaee et al.'s achievability bounds [36] and an analogue of the metaconverse [21]. We omit it here due to space constraints and refer to the full version [32]. Note that the gap between achievable and converse bounds is $\log n$, which leaves room for improvements. In channel coding, the gap is at most $\frac{1}{2} \log n$, and constant for certain channels (see, e.g., [2], [29], [33] for recent work on this topic).

We are in particular interested in the situation where $P_{X Y}$ results from measurements on a channel with (independent) quantum bit error rate $Q$, as it for example occurs in BB84 [3] or the 6-state protocol [5]. Here, we (at least) require $\varepsilon$ correctness for the distribution

$$
\begin{aligned}
& P_{X Y}^{Q}(0,0)=P_{X Y}^{Q}(1,1)=\frac{1-Q}{2}, \quad \text { and } \\
& P_{X Y}^{Q}(0,1)=P_{X Y}^{Q}(1,0)=\frac{Q}{2}
\end{aligned}
$$


The distribution $\left(P_{X Y}^{Q}\right)^{n}$ describes a typical manifestation of two random strings for which the expected bit error rate is $Q$. For the following, we thus say, that a IR protocol is $(\varepsilon, Q)$-correct if it $\varepsilon$-correct on $P_{X Y}^{Q}$. We show the following, specialized bounds:

Corollary 2. Let $0<\varepsilon<1$ and let $0<Q<\frac{1}{2}$. Then, for large $n$, any $(\varepsilon, Q)$-correct IR protocol satisfies

$$
\log |\mathcal{M}| \geq \xi(n, \varepsilon ; Q) \cdot n h(Q)-\frac{1}{2} \log n-O(1), \quad \text { where }
$$

$$
\xi(n, \varepsilon ; Q):=1+\frac{1}{\sqrt{n}} \frac{\sqrt{v(Q)}}{h(Q)} \Phi^{-1}(1-\varepsilon) .
$$

Here, $h(x)=-x \log x-(1-x) \log (1-x)$ and $v(x)=x(1-$ $x) \log ^{2}(x /(1-x))$. Furthermore, there exists a $(\varepsilon, Q)$-correct IR protocol with $\log |\mathcal{M}| \leq \xi(n, \varepsilon ; Q) \cdot n h(Q)+\frac{1}{2} \log n+O(1)$.

The proof of Eq. (2) follows by specializing Theorem 1 to the distribution $P_{X Y}^{Q}$.

Numerical simulations reveal that the approximation in Corollary 2 is very accurate even for small values of $n$. More precisely, we establish an analytical bound, Eq. (3) on the next page, where $F^{-1}(\cdot ; n, p)$ is the inverse of the cumulative distribution function of the binomial distribution. This bound can be evaluated numerically even for reasonably large $n$.

\section{RESULTS}

As shown above, $\log |\mathcal{M}| \approx \xi(n, \varepsilon ; Q) n h(Q)$ is theoretically achievable and optimal up to additive constants. This implies, for example, that the approximation $\log |\mathcal{M}| \approx$ $1.1 n h(Q)$ is provably too optimistic if $\xi(n, \varepsilon ; Q)>1.1$, e.g. for $n<10^{4}, Q=2.5 \%$ and $\varepsilon=10^{-2}$. The function $\xi(\cdot, \varepsilon ; Q)$ is plotted in Fig. 1 for different values of $\varepsilon$ and $Q$. However, theoretical achievability only ensures the existence of a code without actually constructing it; in particular, it is not known if efficient codes used in practical implementations can achieve the above bound. Hence, the approximation given in Corollary 2 is generally too optimistic and must be checked against what can be achieved using state-of-the-art codes.

We suggest that practical information reconciliation codes for finite block lengths should be benchmarked against the fundamental limit for that block length, and not against the asymptotic limit. Moreover, we conjecture that, for some constants $\xi_{1}, \xi_{2} \geq 1$ depending only on the coding scheme used, the leaked information due to information reconciliation can be approximated well by

$$
\text { leak }_{E C} \approx \xi_{1} \cdot n h(Q)+\xi_{2} \cdot \sqrt{n v(Q)} \Phi^{-1}(1-\varepsilon)
$$

for a large range of $n$ and $Q$ as long as $\varepsilon$ is small enough. Here, $\xi_{1}$ measures how well the code achieves the asymptotic limit (1st order) whereas $\xi_{2}$ measures the 2nd order deficiency.

In the following we test this conjecture against some stateof-the-art error correcting codes and find $\xi_{1}$ and $\xi_{2}$ for these codes. Furthermore, we are concerned with the following system design question: given a reconciliation failure probability $\varepsilon$ and block length $n$, what is the leakage expected in practice?

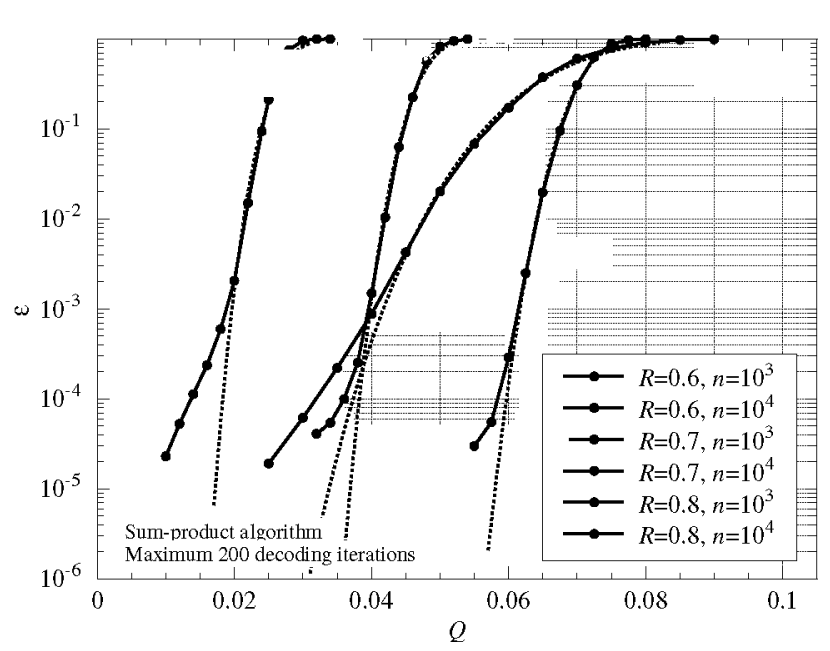

Fig. 2: Simulated block error rates $\varepsilon$ of LDPC codes of length $n=10^{3}$ and $n=10^{4}$ and coding rates $R=0.6, R=0.7$ and $R=0.8$ as a function of quantum bit error rate $Q$.

For this numerical analysis we focus on low-density paritycheck (LDPC) codes following several recent implementations [16], [20], [35].

We constructed a set of LDPC codes with the progressive edge algorithm (PEG) [13] using the following degree polynomials:

$$
\begin{aligned}
\lambda_{1}(x) & =0.1560 x+0.3482 x^{2}+0.1594 x^{13}+0.3364 x^{14} \\
\lambda_{2}(x) & =0.1305 x+0.2892 x^{2}+0.1196 x^{10}+0.1837 x^{12} \\
& +0.2770 x^{14} \\
\lambda_{3}(x) & =0.1209 x+0.2738 x^{2}+0.1151 x^{5}+0.2611 x^{10} \\
& +0.2291 x^{14}
\end{aligned}
$$

where $\lambda_{1}(x), \lambda_{2}(x)$ and $\lambda_{3}(x)$ were designed for coding rates $0.6,0.7$ and 0.8 , respectively [7].

Fig. 2 shows the block error rate of the codes with rates $0.6,0.7,0.8$, and lengths $10^{3}, 10^{4}$ as a function of $Q$. The thick lines connect the simulated points while the dotted lines represent a fit following Eq. (4) (the fit values can be found in Table I). The fit perfectly reproduces the so-called waterfall region of the codes. However, Eq. (4) drops sharply with $Q$ for $Q \in[0,0.1]$ while LDPC codes experience an error floor. In this second region the fit can not approximate the behavior of the codes.

In Fig. 1 we plot the function $\xi(n, \varepsilon ; Q)$ and the efficiency results obtained with LDPC codes. We chose as representative lengths $10^{3}, 10^{4}, 10^{5}$, and $10^{6}$. For every block length we constructed codes of rates $0.6,0.7$ and 0.8 following $\lambda_{1}(x)$, $\lambda_{2}(x)$ and $\lambda_{3}(x)$. The points in the figure were obtained by puncturing and shortening the original codes [16] until the desired block error rate was obtained. The results show an extra inefficiency due to the use of real codes. This inefficiency shares strong similarities with the converse bound, its separation from the asymptotic value is greater for lower values of $Q$, block error rates and lengths and fades as these parameters increase. For example, for $n=10^{4}, Q=1.0 \%$ 


$$
\log |\mathcal{M}| \geq n h(Q)+\left(n(1-Q)-F^{-1}(\varepsilon(1+1 / \sqrt{n}) ; n, 1-Q)-1\right) \log \frac{1-Q}{Q}-\frac{1}{2} \log n-\log \frac{1}{\varepsilon}
$$

and $\varepsilon=10^{-2}$ the extra inefficiency due to the use of real codes is over 1.2 while for $n=10^{6}, Q=5.0 \%$ and $\varepsilon=10^{-1}$ the extra inefficiency is close to 1.05 .

Finally, we address the design question posed above, that is, we study the efficiency variation as a function of the block error rate for fixed $n$ and $Q$. For this setting we need code constructions that allow to modulate the rate with fixed blocklength. The most natural modulating option would have been to construct codes for every $n$ of interest and augment [18] the codes, that is, eliminate some of the restrictions that the codewords verify. However, it is known that LDPC codes do not perform well under this rate adaptation technique [34]. In consequence, we constructed a different code with the PEG algorithm for every rate. In order to obtain a smooth efficiency curve we used the degree polynomials $\lambda_{1}(x), \lambda_{2}(x)$ and $\lambda_{3}(x)$ for constructing all codes even with coding rates different to the design rate.

Fig. 3 shows the efficiency as a function of the block error rate. Each of the two subfigures (a) and (b) show the simulation results for codes of length $10^{3}$ and $10^{4}$, respectively. Colours blue and red correspond to $Q=1.5 \%$ and $3.0 \%$ in subfigure (a) and to $2.5 \%$ and $4.0 \%$ in subfigure (b). The solid lines show the bound given by Corollary 2, similar to Fig. 1 we observe that, ceteris paribus, lower values of $Q$ imply higher values of $\xi$. The points show values achieved by LDPC codes: each point represents the block error rate of a different parity check modulated code. Finally the dotted lines show the best least squares fit to Eq. 4, the values of $\xi_{1}$ and $\xi_{2}$ can be found in Table I. From these curves we can extract some useful design information, 1) if the target failure probability is very high [16] then the gain obtained by increasing the block length is modest, 2) if the target failure probability is low (below $10^{-4}$ ) the leakage is over a fifty percent larger than the optimal one for moderate block lengths and 3) for block-length $10^{5}$, the largest length for which we could compute simulations in the whole block error rate region, we were unable to consistently offer efficiency values below 1.1 and furthermore we report no point with $f$ below 1.05 .

Table I shows the values of $\xi_{1}$ and $\xi_{2}$ used in Figs. 1, 2, and 3 to fit the data points obtained from the simulations. In these curves $\xi_{1}$ is - independently of $\varepsilon, n, Q$-in the range $[1.05,1.16]$ while the 2 nd order deficiency $\xi_{2}$ is more sensible to the parameter variations. For the first four rows, that correspond to Fig. 1 with fixed $Q$ and $\varepsilon, \xi_{2}$ is in the range $[2.41,3.82]$, for the middle six rows, that correspond to Fig. 2 with fixed $n$ and leak, $\xi_{2}$ is in the range $[1.49,1.96]$, while for the last four rows, that correspond to Fig. 3 with fixed $n$ and $Q, \xi_{2}$ is in the range $[1.26,1.58]$. Note that for each scenario, the averages in these ranges could safely be used for system design purposes since necessarily codes with those $\xi_{1}$ and $\xi_{2}$ values or better exist.

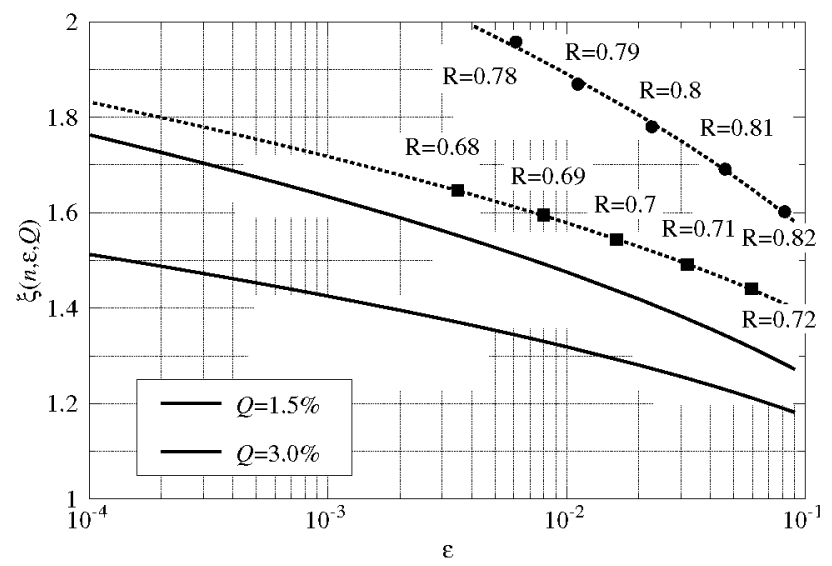

(a) $n=10^{3}$

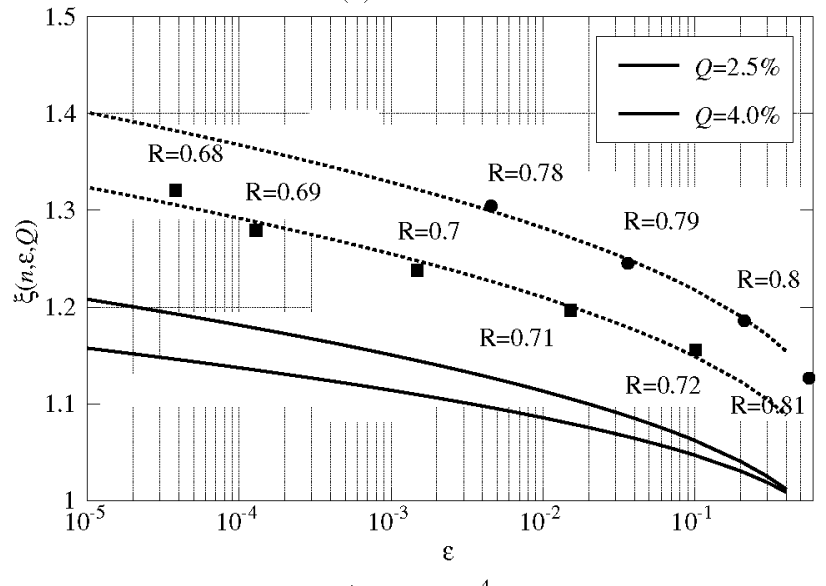

(b) $n=10^{4}$

Fig. 3: Ratio between the leakage and the asymptotical optimum in several scenarios as a function of the block error rate $\varepsilon$. Subfigures (a) and (b) show results for block lengths $10^{3}$ and $10^{4}$, respectively. In each subfigure the solid lines show the converse bound from Corollary 2 while the dotted lines show the values achieved with actual LDPC codes.

\section{CONCLUSION}

In this paper we studied the fundamental limits for information reconciliation in the finite key regime. These limits imply that the commonly used approximation $\log |\mathcal{M}| \approx 1.1 n h(Q)$ is too optimistic for a range of error rates and block-lengths, and proposed a two-parameter approximation that takes into account finite key effects.

We compared the finite length limits with LDPC codes and found a consistent range of achievable finite-length efficiencies. These efficiencies should be of use to the quantum key distribution systems designer. One question that we leave open is the study of these values for different coding families.

Finally, it is clear that PE and PA also contribute to finitelength losses in the QKD key rate. While it seems possible 
TABLE I: Values of $\xi_{1}$ and $\xi_{2}$ for the fitted curves in Fig. 1-3.

\begin{tabular}{cccc|cc}
$n$ & $Q$ & $\varepsilon$ & leak & $\xi_{1}$ & $\xi_{2}$ \\
\hline- & 0.010 & $10^{-2}$ & - & 1.13 & 3.82 \\
- & 0.025 & $10^{-2}$ & - & 1.07 & 3.71 \\
- & 0.050 & $10^{-2}$ & - & 1.06 & 3.54 \\
- & 0.050 & $10^{-1}$ & - & 1.05 & 2.41 \\
\hline $10^{3}$ & - & - & $4 \cdot 10^{2}$ & 1.11 & 1.39 \\
$10^{3}$ & - & - & $3 \cdot 10^{2}$ & 1.12 & 1.45 \\
$10^{3}$ & - & - & $2 \cdot 10^{2}$ & 1.13 & 1.69 \\
$10^{4}$ & - & - & $4 \cdot 10^{3}$ & 1.07 & 1.41 \\
$10^{4}$ & - & - & $3 \cdot 10^{3}$ & 1.08 & 1.44 \\
$10^{4}$ & - & - & $2 \cdot 10^{3}$ & 1.11 & 1.89 \\
\hline $10^{3}$ & 0.015 & - & - & 1.16 & 1.52 \\
$10^{3}$ & 0.030 & - & - & 1.16 & 1.31 \\
$10^{4}$ & 0.025 & - & - & 1.14 & 1.26 \\
$10^{4}$ & 0.040 & - & - & 1.07 & 1.58 \\
\hline
\end{tabular}

to investigate fundamental limits in PA based on the normal approximation of randomness extraction against quantum side information [30] as a separate problem, we would in fact need to investigate it jointly with IR since there is generally a tradeoff between the two tasks that needs to be optimized over.

Acknowledgements: MT thanks N. Beaudry, S. Bratzik, F. Furrer, M. Hayashi, C.C.W. Lim, and V.Y.F. Tan for helpful comments and pointers to related work. MT acknowledges funding from the Ministry of Education (MOE) and National Research Foundation Singapore, as well as MOE Tier 3 Grant "Random numbers from quantum processes" (MOE2012-T31-009). CP has been funded by the Vienna Science and Technology Fund (WWTF) through project ICT10-067 (HiPANQ). DE would like to acknowledge support from CHIST-ERA project Composing Quantum Channels, Project No. PRIPIMCHI-2011-1071.

\section{REFERENCES}

[1] S. Abruzzo, H. Kampermann, M. Mertz, and D. Bruß. Quantum key distribution with finite resources: Secret key rates via Rényi entropies. Phys. Rev. A, 84(3):032321, 2011.

[2] Y. Altug and A. B. Wagner. The Third-Order Term in the Normal Approximation for Singular Channels. 2013. arXiv: 1309.5126.

[3] C. H. Bennett and G. Brassard. Quantum Cryptography: Public Key Distribution and Coin Tossing. In Proc. IEEE Int. Conf. Comp., Sys. Signal Process., pages 175-179, Bangalore, 1984. IEEE.

[4] S. Bratzik, M. Mertz, H. Kampermann, and D. Bruß. Min-entropy and quantum key distribution: Nonzero key rates for small numbers of signals. Phys. Rev. A, 83(2), 2011.

[5] D. Bruß. Optimal Eavesdropping in Quantum Cryptography with Six States. Phys. Rev. Lett., 81(14):3018-3021, 1998.

[6] R. Y. Q. Cai and V. Scarani. Finite-key Analysis for Practical Implementations of Quantum Key Distribution. New J. Phys., 11(4):045024, 2009.

[7] S.-Y. Chung, J. Forney G.D., T. J. Richardson, and R. Urbanke. On the Design of Low-Density Parity-Check Codes Within $0.0045 \mathrm{~dB}$ of the Shannon Limit. IEEE Commun. Lett., 5(2):58-60, 2001.

[8] A. K. Ekert. Quantum Cryptography Based on Bell's Theorem. Phys. Rev. Lett., 67(6):661-663, 1991.

[9] M. Hayashi. Practical Evaluation of Security for Quantum Key Distribution. Phys. Rev. A, 74(2), 2006.

[10] M. Hayashi. Second-Order Asymptotics in Fixed-Length Source Coding and Intrinsic Randomness. IEEE Trans. Inf. Theory, 54(10):4619-4637, 2008.

[11] M. Hayashi. Information Spectrum Approach to Second-Order Coding Rate in Channel Coding. IEEE Trans. Inf. Theory, 55(11):4947-4966, 2009.
[12] M. Hayashi and T. Tsurumaru. Concise and Tight Security Analysis of the Bennett-Brassard 1984 Protocol with Finite Key Lengths. New $J$. Phys., 14(9):093014, 2012.

[13] X.-Y, Hu, E. Eleftheriou, and D.-M. Arnold. Regular and Irregular Progressive Edge-Growth Tanner Graphs. IEEE Trans. Inf. Theory, 51(1):386-398, 2005

[14] C. C. W. Lim, C. Portmann, M. Tomamichel, R. Renner, and N. Gisin. Device-Independent Quantum Key Distribution with Local Bell Test. Phys. Rev. X, 3(3):031006, 2013

[15] H.-K. Lo, H. Chau, and M. Ardehali. Efficient Quantum Key Distribution Scheme and a Proof of Its Unconditional Security. J. Cryptol., 18(2):133-165, 2004.

[16] J. Martinez-Mateo, D. Elkouss, and V. Martin. Key Reconciliation for High Performance Quantum Key Distribution. Sci. Rep., 3(1576):1-6, 2013.

[17] D. Mayers. Unconditional Security in Quantum Cryptography. J. ACM, 48(3):351-406, 2001.

[18] R. H. Morelos-Zaragoza. The Art of Error Correcting Coding, John Wiley and Sons Inc, 2006.

[19] T. Moroder, M. Curty, and N. Lütkenhaus, One-Way Quantum Key Distribution: Simple Upper Bound on the Secret Key Rate. Phys. Rev. A, 74(5):052301, 2006.

[20] C. Pacher, G. Lechner, C. Portmann, O. Maurhart, and M. Peev. Efficient QKD Postprocessing Algorithms, 2012. Available online: https://sqt.ait.ac.at/software/attachments/download/504.

[21] Y. Polyanskiy, H. V. Poor, and S. Verdú. Channel Coding Rate in the Finite Blocklength Regime. IEEE Trans. Inf. Theory, 56(5):2307-2359, 2010.

[22] R. Renner. Security of Quantum Key Distribution. PhD thesis, ETH Zurich, 2005. arXiv: quant-ph/0512258.

[23] V. Scarani, H. Bechmann-Pasquinucci, N. Cerf, M. Dušek, N. Lütkenhaus, and M. Peev, The Security of Practical Quantum Key Distribution, Rev. Mod. Phys., 81(3):1301-1350, 2009.

[24] V. Scarani and R. Renner. Quantum Cryptography with Finite Resources: Unconditional Security Bound for Discrete-Variable Protocols with OneWay Postprocessing. Phys. Rev. Lett., 100(20), 2008.

[25] P. W. Shor and J. Preskill. Simple Proof of Security of the BB84 Quantum Key Distribution Protocol. Phys. Rev. Lett., 85(2):441-444, 2000.

[26] D. Slepian and J. Wolf. Noiseless Coding of Correlated Information Sources. IEEE Trans. Inf. Theory, 19(4):471-480, 1973.

[27] V. Strassen. Asymptotische Abschätzungen in Shannons Informationstheorie, In Trans. Third Prague Conf. Inf. Theory, pages 689-723, Prague, 1962.

[28] V. Y. F. Tan and O. Kosut. The Dispersion of Slepian-Wolf Coding. In Proc. IEEE ISIT, 2012

[29] V. Y. F, Tan and M. Tomamichel. The Third-Order Term in the Normal Approximation for the AWGN Channel. 2013. arXiv: 1311.2337.

[30] M. Tomamichel and M. Hayashi. A Hierarchy of Information Quantities for Finite Block Length Analysis of Quantum Tasks. IEEE Trans. Inf. Theory, 59(11):7693-7710, 2013.

[31] M. Tomamichel, C. C. W. Lim, N. Gisin, and R. Renner. Tight FiniteKey Analysis for Quantum Cryptography. Nat. Commun., 3:634, 2012.

[32] M. Tomamichel, J. Martinez-Mateo, C. Pacher, and D. Elkouss. Fundamental Finite Key Limits for Information Reconciliation in Quantum Key Distribution. 2014, arXiv: 1401.5194.

[33] M. Tomamichel and V. Y. F. Tan. A Tight Upper Bound for the ThirdOrder Asymptotics for Most Discrete Memoryless Channels. IEEE Trans. Inf. Theory, 59(11):7041-7051, 2013.

[34] D. Varodayan, A. Aaron, and B. Girod. Rate-Adaptive Codes for Distributed Source Coding. Signal Processing, 86(11):3123-3130, 2006.

[35] N. Walenta, A. Burg, D. Caselunghe, J. Constantin, N. Gisin, O. Guinnard, R. Houlmann, P. Junod, B. Korzh, N. Kulesza, M. Legré, C. C. W. Lim, T. Lunghi, L. Monat, C. Portmann, M. Soucarros, P. Trinkler, G. Trolliet, F. Vannel, and H. Zbinden. A Fast and Versatile QKD System With Hardware Key Distillation and Wavelength Multiplexing. 2013. arXiv: 1309.2583.

[36] M. H. Yassaee, M. R. Aref, and A. Gohari. A Technique for Deriving One-Shot Achievability Results in Network Information Theory. In Proc. IEEE ISTT, 2013. 